\title{
Fetal aortic wall thickness: a marker of hypertension in IUGR children?
}

\begin{abstract}
Vincenzo Zanardo, Silvia Visentin, Daniele Trevisanuto, Martina Bertin, Francesco Cavallin and Erich Cosmi
Fetuses with intrauterine growth restriction (IUGR) have significant aortic intima-media thickening (aIMT), which suggests that preclinical atherosclerosis might predispose the infants to hypertension. However, the natural course of alMT in babies with IUGR remains an open question. The study enrolled 77 pregnant women between January 2007 and August 2009. The fetuses were classified as AGA (appropriate for gestational age) or IUGR, if the estimated fetal weight was between the 10th and 90th percentile or below the 10th percentile (with umbilical artery pulsatility index (PI) $>2$ s.d.), respectively. Anthropometric parameters and aIMT were detected in each IUGR and AGA fetus at a mean gestational age of 32 weeks. The follow-up was performed in 25 IUGR and 25 AGA infants at a mean postnatal age of 18 months; the previous measurements were repeated, and blood pressure measurements were taken. The maximum aIMT was significantly higher in the IUGR fetuses and infants compared with the AGA infants, both in utero $(2.05 \pm 0.43$ vs. $1.05 \pm 0.19 \mathrm{~mm}, P<0.001)$ and at the follow-up $(2.3 \pm 0.8$ vs. $1.06 \pm 0.18 \mathrm{~mm}, P<0.0001)$, the resulting values significantly correlated $(P=0.018)$ with one another. The systolic blood pressure was significantly increased in the IUGR subjects $(123 \pm 16$ vs. $104 \pm 8.5 \mathrm{~mm} \mathrm{Hg}, P<0.0004)$, and it correlated with the prenatal and postnatal aIMT values $(P<0.0156$ and $P<0.0054$, respectively). The aortic wall thickening progression in IUGR fetuses and infants differed from AGA, which may predispose the infants to hypertension early in life and cardiovascular risk later in life.
\end{abstract}

Hypertension Research (2013) 36, 440-443; doi:10.1038/hr.2012.219; published online 31 January 2013

Keywords: blood pressure; fetal arterial wall thickness; IUGR fetuses and infants; preclinical atherosclerosis

\section{INTRODUCTION}

Low birth weight is caused either by preterm birth or by intrauterine growth restriction (IUGR), or a combination of both, and has been associated with higher blood pressure (BP) in childhood, adolescence and adulthood in a large number of epidemiological and clinical studies on cardiovascular risk. ${ }^{1-6}$ High BP is part of the metabolic syndrome, and because associated diseases, such as atherosclerosis, coronary heart disease and stroke, have also been linked to low birth weight, it is generally conceived that these conditions share antenatal risk factors. ${ }^{7,8}$ This fact is relevant, considering that low birth weight is a complication in $\sim 10 \%$ of all pregnancies in the Western world. There is an urgent need for further data concerning the outcomes of children suffering from high BP and who have histories of prematurity and poor fetal growth. ${ }^{9}$

The mechanisms whereby slowed intrauterine growth confers vascular risk are not clearly understood, particularly whether fetal events might result in any arterial abnormalities early in the intrauterine life. ${ }^{10-12}$ The earliest pathological evidence of atherosclerosis is apparent in the abdominal aorta by early childhood, and Skilton et al. ${ }^{13,14}$ have described the ultrasoundbased measurement of aortic intima-media thickness in neonates as a feasible, accurate and sensitive marker of atherosclerosis risk. Newborn babies with growth restriction were found to have significant aortic thickening, which suggested that prenatal events might predispose these infants to later cardiovascular risk. ${ }^{15,16}$ Therefore, early endothelial dysfunction and intima-media thickening may significantly contribute to the premature stiffening of the aortic tree, which ultimately predisposed these individuals to hypertension. ${ }^{17,18}$ However, the natural course of aortic intima-media thickening in small infants still remains an open question.

During a recent longitudinal prospective study to evaluate aortic wall thickness in intrauterine growth restricted fetuses and infants, ${ }^{12}$ we planned additional analyses to determine whether aortic thickening was predictive of higher BP during infancy. Therefore, the aim of the present study was twofold: (i) to measure in utero and postnatally at a mean follow-up of 18 month of life the abdominal aortic intima-media thickness (aIMT) and BP in IUGR and adequate for gestational age (AGA) fetuses; and (ii) to assess the correlation between aIMT and BP development to test the hypothesis of the existence of vascular dysfunction in IUGR fetuses and children, and determine whether the fetal aortic thickening in IUGR infants required surveillance for cardiovascular risk in childhood. 


\section{METHODS}

This secondary prospective analysis of IUGR patients was performed as part of a multicenter collaboration. ${ }^{19}$ In the original study, the patients were enrolled if they met the following inclusion criteria: (I) singleton pregnancy; (II) gestational age determined by the last menstrual period and confirmed by sonography $<20$ weeks and (III) IUGR diagnosed by an abdominal circumference $<5$ th percentile, elevation of the umbilical artery and a umbilical artery pulsatility index $(\mathrm{PI})>2$ s.d.

For this analysis, we evaluated a subset of IUGR and AGA patients in utero and at the follow-up; these patients had at least three Doppler examinations before delivery at the Institute of Obstetrics and Gynecology of Padua University, Padua, Italy, between January 2007 and August 2009. Written informed consent was obtained from each woman before the enrollment, and the project was approved by the University Hospital Committee for Research on Human subjects. The women included in the study were all between 24 and 30 weeks of gestation. The data concerning the women and their pregnancies were recorded according to the established procedures of our Department. The maternal smoking status (current smoker, yes or no) was obtained from the medical history. The inclusion criteria at admission were single pregnancy and gestational age determination from a combination of maternal menstrual dating and obstetrical ultrasound at $<20$ weeks. The exclusion criteria were a twin pregnancy, major congenital anomalies, pregnancies complicated by maternal history of cardiovascular disease or endocrine disorders such as diabetes, hypercholesterolemia, pre-eclampsia, thyroid or adrenal problems, clinical chorioamnionitis or perinatal infections. Additionally, mothers who had received alcohol or drugs, such as ritodrine and corticosteroids (except for fetal lung maturation) were excluded. The fetuses were classified as AGA or IUGR if the estimated fetal weight was between the 10th and 90th percentile or below the 10th percentile, respectively, with Doppler velocimetry abnormalities (umbilical artery, PI $>2$ s.d.). ${ }^{20,21}$ Standard tables for singleton pregnancies were used to define the fetal weight percentiles for the gestational ages. ${ }^{22}$ Thirty-four IUGR fetuses met the inclusion criteria. In nine cases, the parents declined consent. Therefore, 25 fetuses with IUGR were included in the study, and the control group comprised 25 consecutive healthy and AGA fetuses. For each matched fetus, we recorded the aIMT and anthropometrical and sonographic features. ${ }^{12}$ The follow-up ultrasound aIMT was performed in the IUGR and AGA children at a mean postnatal age of 18 months, and included the anthropometrical and sonographic parameters and BP measurements.

The BP measurements were performed using a standard sphygmomanometer (Philip Medical System Monitor, Agilent, M3046A model, M4, Boebingen, Germany) with a cuff size that was adapted to the subject's right arm circumference. Three independent measurements for each participant were taken; the arithmetic mean was considered for the study, as proposed by current guidelines. ${ }^{23}$

The aorta intima-media thickness was measured with a high-resolution ultrasound scan using an ultrasound machine (Antares, Siemens Medical Solutions, Mountain View, CA, USA; Voluson 530 Expert, GE Medical Systems, Milwaukee, WI, USA), equipped with $3,5-5 \mathrm{Mhz}$ linear array transducer. The IMT was measured at the dorsal arterial wall of the aorta (in a straight, nonbranched $1 \mathrm{~cm}$ longitudinal segment of the proximal abdominal aorta), as previously described. ${ }^{12}$ The intima-media thickness was calculated with validated edge detection software, which was previously shown to be accurate and reproducible. ${ }^{12}$ This study was conducted with the approval of the Padua Hospital Research Ethics Committee

\section{Statistical analysis}

The continuous data were expressed as the means \pm s.d., whereas the categorical data were expressed as number (\%). The continuous and categorical data were compared using the Kruskal -Wallis's test and Fischer's exact test. A $P$-value $<0.05$ was considered significant. The statistical analyses were performed using software package SPSS 17 (SPSS, Chicago, IL, USA).

\section{RESULTS}

The anthropometrical, sonographic and clinical characteristics of the study population are shown in Table 1 .
Table 1 Anthropometric, sonographic, and clinical measurements among IUGR and AGA fetuses and neonates. Mean \pm s.d.

\begin{tabular}{lccc}
\hline & IUGR & AGA & \\
Number, n; percent (\%) & $\mathrm{n}=25$ & $\mathrm{n}=25$ & $\mathrm{P}$ \\
\hline Mothers: & & & \\
Age (y) & $30.5( \pm 11)$ & $31.2( \pm 10)$ & 0.42 \\
Smoking & $3(12)$ & $7(28)$ & 0.33 \\
Nulliparous & $13(54)$ & $15(60)$ & 0.93 \\
Cesarean delivery & $16(64)$ & $10(40)$ & 0.14 \\
Vaginal delivery & $9(36)$ & $15(60)$ & 0.14 \\
& & & \\
Fetal measurements: & & & 0.96 \\
Gestational age (weeks) & $32.1+1.4$ & $32+1.9$ & 0.01 \\
Abdominal circumference (mm) & $278 \pm 12$ & $288 \pm 18$ & 0.0002 \\
Estimated fetal weight (g) & $1899 \pm 300$ & $2255 \pm 268$ & $<0.001$ \\
alMT (mm) & $2.05 \pm 0.43$ & $1.05 \pm 0.19$ & \\
& & & \\
Neonatal measurements: & & & 0.0001 \\
Gestational age (w) & $33 \pm 1$ & $37 \pm 1.2$ & 0.0001 \\
Male/Female & $13 / 12$ & $9 / 16$ & \\
Birth weight (g) & $1940 \pm 290$ & $2980 \pm 160$ & $<0.014$ \\
\hline
\end{tabular}

Abbreviations: AGA, adequate for gestational age; alMT, aortic intima-media thickening, IUGR, intrauterine growth restriction. P: statistical significance at $P<0.05$.

We found no differences for the measurements with respect to the maternal age at delivery, parity, nicotine abuse or the delivery mode. No gender disparity was encountered comparing the IUGR vs. AGA. The mean birth weight of the IUGR children born was $1940 \mathrm{~g}$ at a mean gestational age of 33 weeks. The estimated fetal weight at the time of the aIMT measurement was $1899 \mathrm{~g}$, and the abdominal circumference was $278 \mathrm{~mm}$. The AGA neonates had a mean gestational duration of 37 weeks and a mean birth weight of $2980 \mathrm{~g}$. At the moment of the study, the estimated fetal weight was $2255 \mathrm{~g}$, and the abdominal circumference was $287 \mathrm{~mm}$. The mean aIMT for the fetuses at 32 gestational weeks, $n$ was significantly different between the IUGR fetuses and AGA $(2.3 \pm 0.43$ vs. $1.05 \pm 0.19 \mathrm{~mm}, P<0.001)$.

Likewise, a significant negative correlation was found between aIMT and estimated fetal weight at 32 gestational weeks in the IUGR fetuses $(P<0.003, r=-0.58)$; this negative correlation indicated a trend of increasing aortic thickness with the severity of intrauterine growth retardation. There was no significant correlation with the AGA fetuses $(P=0.3, r=-0.2)$, as shown in Table 3 .

At the follow-up (Table 2), the mean body weight for the IUGR children was $12300 \pm 1200 \mathrm{~g}$ and $12300 \pm 1100 \mathrm{~g}$ for the AGA children. A comparison between the two groups revealed a higher prevalence of body weight $\geqslant 75^{\circ}$ percentile in the IUGR children (11 vs. $3, P<0.02)$.

The mean aIMT at the postnatal age of 18 months was $2.3 \pm 0.8 \mathrm{~mm}$ for the IUGR children. In contrast, the children born with AGA had a mean value of infant aIMT of $1.06 \pm 0.18 \mathrm{~mm}$, which showed that the aortic wall thickness was significantly increased in the neonates with IUGR compared with the AGA neonates $(P<0.0001)$.

Moreover, a positive linear correlation was observed between the prenatal aIMT values and the postnatal aIMT values $(P<0.0185$, $r=0.48)$ for the children born with IUGR, a phenomenon that was not observed in the AGA children (Table 3 ).

Both the mean pulse BP $(84 \pm 9.5$ vs. $86 \pm 12 \mathrm{~mm} \mathrm{Hg}, P=0.8)$ and the diastolic BP $(65 \pm 7.4$ vs. $65 \pm 6 \mathrm{~mm} \mathrm{Hg}, P=0.68)$ were not 
Table 2 Anthropometric, sonographic and clinical measurements among IUGR and AGA infants at the mean postnatal age of 18 months follow up

\begin{tabular}{lccc}
\hline & IUGR & AGA & \\
Follow up & $\mathrm{n}=25$ & $\mathrm{n}=25$ & $\mathrm{P}$ \\
\hline Postnatal age (months) & $18.5 \pm 6$ & $20.2 \pm 7.5$ & 0.63 \\
Body weight (g) & $12300 \pm 1200$ & $12300 \pm 1100$ & 0.75 \\
Infant alMT (mm) & $2.3 \pm 0.8$ & $1.06 \pm 0.18$ & $<0.0001$ \\
Systolic BP (mm Hg) & $123 \pm 16$ & $104 \pm 8.5$ & 0.0004 \\
Diastolic BP (mm Hg) & $65 \pm 7.4$ & $65 \pm 6$ & 0.68 \\
Pulse pressure (mm Hg) & $84 \pm 9.5$ & $86 \pm 12$ & 0.8 \\
\hline
\end{tabular}

Abbreviations: AGA, adequate for gestational age; alMT, aortic intima-media thickening

$\mathrm{BP}$, blood pressure; IUGR, intrauterine growth restriction.

$P$ : statistically significant at $P<0.05$.

Table 3 Correlations between aIMT in utero and aIMT and/or blood pressure among IUGR and AGA fetuses and infants at the mean follow-up postnatal age of 18 months

\begin{tabular}{lll}
\hline & $r$ & \\
\hline IUGR: aIMT in utero vs. alMT at follow up & 0.48 & 0.01 \\
AGA: aIMT in utero vs. alMT at follow up & 0.26 & 0.1 \\
IUGR: aIMT in utero vs. systolic BP & 0.49 & 0.01 \\
AGA: aIMT in utero vs. systolic BP & -0.009 & 0.9 \\
IUGR: aIMT in utero and EFW & -0.58 & 0.003 \\
AGA: aIMT in utero and EFW & -0.2 & 0.3 \\
IUGR: aIMT at follow up vs. systolic BP & 0.59 & 0.005 \\
AGA: alMT at follow up vs. systolic BP & -0.073 & 0.7 \\
\hline
\end{tabular}

Abbreviations: AGA, adequate for gestational age; alMT, aortic intima-media thickening, $\mathrm{BP}$, blood pressure; EFW, estimated fetal weight; IUGR, intrauterine growth restriction. $P$ : statistically significant at $P<0.05$.

significantly different between the infants born with IUGR and those born with AGA. A comparison between the two groups revealed higher systolic BP in the IUGR children (123 \pm 16 vs. $104 \pm 85$; $P<0.0004$ ), as shown in Table 2.

In addition, systolic BP was positively correlated with both prenatal $(P<0.0156, r=0.49)$ and postnatal aIMT $(P<0.0054, r=0.59)$ in the IUGR infants. There was no correlation between systolic BP and aortic wall thickness in the AGA children (Table 3).

\section{DISCUSSION}

Certain prenatal factors may affect neonate blood pressure, but the existing evidence is inconclusive and the mechanisms behind these factors are unclear. We examined the mediating roles of IUGR in the associations between childhood BP and fetal and infant aIMT, a marker of preclinical atherosclerosis. ${ }^{13-16}$ The present data revealed that the aortic wall thickness was significantly increased in the fetuses with IUGR and Doppler velocimetry abnormalities compared with the fetuses with AGA. Even when measured at the mean postnatal age of 18 months, the values were still significantly correlated. In addition, systolic BP was significantly increased in the IUGR subjects, with levels significantly correlated to the prenatal and postnatal aIMT values.

Increasing evidence reveals an inverse association between birth weight and blood pressure in children, adolescents and adults, ${ }^{1}$ which provides a new perspective for hypertension etiology and prevention. ${ }^{2}$ However, birth size may just be a marker of other genetic and environmental factors that are the true etiological factors for hypertension. ${ }^{23}$ Therefore, in the present analysis, we expanded our focus beyond fetal weight or birth weight, and instead focused on fetal aIMT to examine the prenatal exposures that might program BP in children. ${ }^{13-18}$ This marker of endothelial dysfunction and preclinical atherosclerosis (with increased hypertension and cardiovascular risk as an adult) was chosen because the clinical evidence has linked aIMT, and the low birth weight caused either by preterm birth, intrauterine growth retardation or a combination of the two. ${ }^{24,25}$

Furthermore, in addition to the postnatal events, atheromatous changes have been documented histopathologically in early childhood, particularly in the abdominal aorta, ${ }^{16}$ and it is now possible, thanks to external ultrasonography, to measure the aortic wall thickness in vivo during fetal ${ }^{12}$ and early postnatal life $^{13}$ in an accurate and reproducible manner. Moreover, the ultrasound measurement of aIMT in IUGR fetuses was found to be a sensitive marker of hypertension in young children and of atherosclerosis risk in adults, ${ }^{11}$ which supported the epidemiological link between impaired fetal growth and later cardiovascular disease risk. ${ }^{16,24,25}$

Although the mechanisms, which underlie these associations remain unclear, these data represent another step in understanding IUGR and the related cardiovascular disease risk. Further studies should focus on additional factors that impact on the early endothelial dysfunction, ${ }^{10}$ in addition to placenta-induced developmental disorders and Doppler umbilical artery vasculopathy. More importantly, the biological mechanisms through, which these prenatal factors are associated with offspring BP remain unclear. Poor knowledge of these mechanisms is a critical barrier for causal interpretation, as well as safe and effective intervention. We hypothesized that IUGR and fetal aIMT have important roles in these mechanisms. Given the associations between aIMT and BP, as well as between birth size and offspring $\mathrm{BP}$, the effects of prenatal factors on offspring BP may be partially explained by persistent aortic wall thickness in IUGR children. However, no studies have systematically examined the mediating roles of IUGR and aIMT in the associations between this prenatal factor and offspring BP.

This is the first study to examine the potential mediation of both IUGR and prenatal aortic stiffening and developing BP. Repeated aIMT measures facilitated the identification of the critical growth periods (fetal, infancy and early childhood) that impact offspring BP. Adjusting for maternal and family factors can remove substantial confounding. Despite the standard protocol, the single measure of blood pressure could introduce measurement errors. We could not distinguish between the impacts of the different components and the rates of both fetal or maternal pregnancy weight gain on infant BP. We had no information on the pregnancy diets and the children's own lifestyle (for example, breastfeeding or formula adoption, diet and salt intake). Despite clear temporality (prenatal aortic stiffening exposures affected IUGR fetuses, which affected childhood BP), the observed associations can be interpreted as causal pathways only by assuming no residual confounding, which is nearly impossible for observational studies.

In conclusion, our new findings can advance current poor knowledge on mechanisms for fetal programming of blood pressure. Evidence from this analysis indicates that, instead of limiting our focus on fetal or neonatal size, monitoring in utero aortic wall thickness may be more important for long-term cardiovascular health. Persistent aortic thickening may predispose patients to later cardiovascular risk, and may influence vasomotor tone and arterial compliance from early in life. 


\section{CONFLICT OF INTEREST}

The authors declare no conflict of interest.

\section{ACKNOWLEDGEMENTS}

This study was conducted with the approval of the Padua Hospital Research Ethics Committee.

1 Johansson S, Iliadou A, Bergvall N, Tuvemo T, Norman M, Cnattingius S. Risk of high blood pressure among young men increases with the degree of immaturity at birth. Circulation 2005; 112: 3430-3436.

2 Huxley RR, Shiell AW, Law CM. The role of size at birth and postnatal catch-up growth in determining systolic blood pressure: a systematic review of the literature. J Hypertens 2000; 18: 815-831.

3 Barker DJ, Bagby SP, Hanson MA. Mechanisms of disease: in utero programming in the pathogenesis of hypertension. Nat Clin Pract Nephrol 2006; 2: 700-707.

4 Barker DJ, Bull AR, Osmond C, Simmonds SJ. Fetal and placental size and risk of hypertension in adult life. BMJ 1990; 301: 259-262.

5 Law C, de Swiet M, Osmond C, Fayers PM, Barker DJ, Cruddas AM, Fall CH. Initiation of hypertension in utero and its amplification throughout life. BMJ 1993; 306: 24-27.

6 Belfort MB, Rifas-Shiman SL, Rich-Edwards J, Kleinman KP, Gillman MW. Size at birth, infant growth, and blood pressure at three years of age. J Pediat 2007; 151: 670-674.

7 Byrne CD, Phillips DI. Fetal origins of adult disease: epidemiology and mechanisms. J Clin Pathol 2005; 3: 822-828.

8 Gluckman PD, Hanson MA. Living the past: evolution, development, and patterns of disease. Science 2004; 305: 1733-1736.

9 Gortner L. Intrauterine growth restriction and risk for arterial hypertension: a causal relationship? J Perinat Med 2007; 35: 361-365.

10 Wen X, Triche EW, Hogan JW, Shenassa ED, Buka SL. Prenatal factors for childhood blood pressure mediated by intrauterine and/or childhood growth? Pediatrics 2011; 127: e713-e721.

11 Dawson JD, Sonka M, Blecha MB, Lin W, Davis PH. Risk factors associated with aortic and carotid intima-media thickness in adolescents and young adults: the Muscatine Offspring Study. J Am Coll Cardiol 2009; 53: 2273-2279.

12 Cosmi E, Visentin S, Fanelli T, Mautone AJ, Zanardo V. Aortic intima media thickness in intrauterine growth restricted fetuses and infants: a longitudinal prospective study. Obstet Gynecol 2009; 114: 1109-1114.
13 Skilton MR, Evans N, Griffiths KA, Harmer JA, Celermajer DS. Aortic wall thickness in newborns with intrauterine growth restriction. Lancet 2005; 365: 1484-1486.

14 Skilton MR. Intrauterine risk factors for precocious atherosclerosis. Pediatrics 2008; 121: $570-574$

15 Koklu E, Kurtoglu S, Akcakus M, Koklu S, Buyukkayhan D, Gumus H, Yikilmaz A. Increased aortic intima-media thickness is related to lipid profile in newborns with intrauterine growth restriction. Horm Res 2006; 65: 269-275.

16 Lo Vasco VR, Salmaso R, Zanardo V, Businaro R, Visentin S, Trevisanuto D, Cosmi E. Fetal aorta wall inflammation in ultrasound-detected aortic intima/media thickness and growth retardation. J Reprod Immunol 2011; 91: 103-107.

17 Doyle LW, Faber B, Callanan C, Morley R. Blood pressure in late adolescence and very low birth weight. Pediatrics 2003; 111: 252-257.

18 Franco MCP, Christofalo DMJ, Sawaya AL, Ajzen SA, Sesso R. Effects of low birth weight in 8- to 13-year-old children: implications in endothelial function and uric acid levels. Hypertension 2006; 48: 45-50.

19 Baschat AA, Cosmi E, Bilardo CM, Berg C, Rigano S, Germer U, Germer U, Moyano D, Turan S, Hartung J, Bhide A, Müller T, Bower S, Nicolaides KH, Thilaganathan B, Gembruch U, Ferrazzi E, Hecher K, Galan HL, Harman CR. Predictors of neonatal outcome in early-onset placental dysfunction. Obstet Gynecol 2007; 109: 253-261.

20 Committee on Practice Bulletins-Gynecology. American College of Obstetricians and Gynecologists, Washington, DC 20090-6920,USA. Intrauterine growth restriction. Clinical management guidelines for obstetrician-gynecologists. Int J Gynaecol Obstet 2001; 72: 85-96.

21 Turan OM, Turan S, Berg C, Gembruch U, Nicolaides KH, Harman CR, Baschat AA. Duration of persistent abnormal ductus venosus flow and its impact on perinatal outcome in fetal growth restriction. Ultrasound Obstet Gynecol 2011; 38: 295-302.

22 Hadlock FP, Harrist RB, Sharman RS, Deter RL, Park SK. Estimation of fetal weight with the use of head, body, and femur measurement-a prospective study. Am J Obstet Gynecol 1985; 151: 333-337.

23 National High Blood Pressure Education Program Working Group on High Blood Pressure in Children and Adolescents. The Fourth report on diagnosis, evaluation, and treatment of high blood pressure in children and adolescents. Pediatrics 2004; 114: 555-576.

24 Järvisalo MJ, Jartti L, Näntö-Salonen K, Irjala K, Rönnemaa T, Hartiala JJ, Raitakari OT. Increased aortic intima-media thickness: a marker of preclinical atherosclerosis in high-risk children. Circulation 2001; 104: 2943-2947.

25 Litwin M, Niemirska A. Intima-media thickness measurements in children with cardiovascular risk factors. Pediatr Nephrol 2008; 24: 707-719. 